\title{
Hier sind Hypoglykämien besonders gefährlich
}

\author{
Die kardiale autonome diabetische Neuropathie \\ (KADN) ist eine häufige Komplikation des Diabetes \\ mellitus mit enormer prognostischer Bedeutung. \\ Sie tritt auch schon im prädiabetischen Stadium auf. \\ Hypoglykämien sollten bei Betroffenen unbedingt \\ vermieden werden, da die Gefahr für Herzrhythmus- \\ störungen dann besonders stark ansteigt.
}

Diabetische Neuropathien betreffen die dicken, myelinisierten Nervenfasern wie die kleinen, wenig oder nicht myelinisierten, darunter die autonomen Nervenfasern. „Diese Small-FiberNeuropathie geht der Schädigung der bemarkten Nervenfasern voraus,“ so PD Dr. Kornelia Konz, Deutsche Klinik für Diagnostik, Wiesbaden. Lange habe man gedacht, die autonome Neuropathie sei eine Spätkomplikation des Diabetes, aber sie trete sehr früh im Verlauf auf. Autonome Neuropathien betreffen viele Organe und Systeme, die mit autonomen Fasern versorgt werden. Dabei nimmt das Herz eine Sonderstellung ein, da es sehr früh und oft betroffen ist. „Daher gilt die KADN auch als Leiterkrankung der autonomen Neuropathien“ so Konz.

\section{Frühe Manifestation}

Von einer KADN sind Typ-1- wie Typ-2-Diabetiker etwa gleich häufig betroffen: Der Unterschied liegt darin, dass die Prävalenz bei Typ-2-Diabetes deutlich mit der Krankheitsdauer zunimmt. Bei symptomatischer KADN beträgt die 5-Jahres-Mortalitätsrate über 50\%. Als Ursache wird ein Substratüberangebot diskutiert, in Form von Glukose und bei Typ-2-Diabetes auch durch ein erhöhtes Angebot an freien Fettsäuren. Dieses Überangebot ist Folge der Insulinresistenz bzw. des absoluten Insulinmangels. Bei Typ-2-Diabetes ist dieses Überangebot bereits im Stadium des metabolischen Syndroms noch vor der Diabetesmanifestation vorhanden. „Daher nehmen die KADN auch mit jedem einzelnen Faktor, den wir unter dem metabolischen Syndrom subsummieren, zu“, so Konz. Im Gegensatz zur den peripheren Neuropathie gibt es keine geeigneten Screeningverfahren für KADN. Man ist auf eine genaue Befragung der Patienten und deren Schilderung der Symptome angewiesen. Doch die Symptome sind wenig spezifisch und sensitiv. Daher handelt es sich bei KADN praktisch immer um eine Ausschlussdiagnose.

Frühes Zeichen einer Schädigung ist eine verminderte Herzfrequenzvariabilität (HFV). Dies bedeutet, dass die Fähigkeit, die Frequenz des Herzrhythmus je nach Bedarf anzupassen, beeinträchtigt ist. Die HFV ist eine Stellgröße des Parasympathikus. Konz betonte: „Die Anfangsstörungen - das sind die eingeschränkte Herzfrequenzvariation, die fixierte Ruhetachykardie, die gestörte zirkadiane Rhythmik von Frequenz und Blutdruck - wird vom Patienten gar nicht bemerkt. Die können Sie nicht erfragen. Sportlich orientierte Patienten klagen evtl. über eine gewisse Belastungsintoleranz." Fortgeschrittene Stadien der KADN gehen mit einer Ruhetachykardie (v.a. Vagusläsion) und orthostatischer Hypotonie (hpts. Sympathikusläsion) einher. Konz betonte: „Was die Patienten bemerken, sind die orthostatischen Hy-

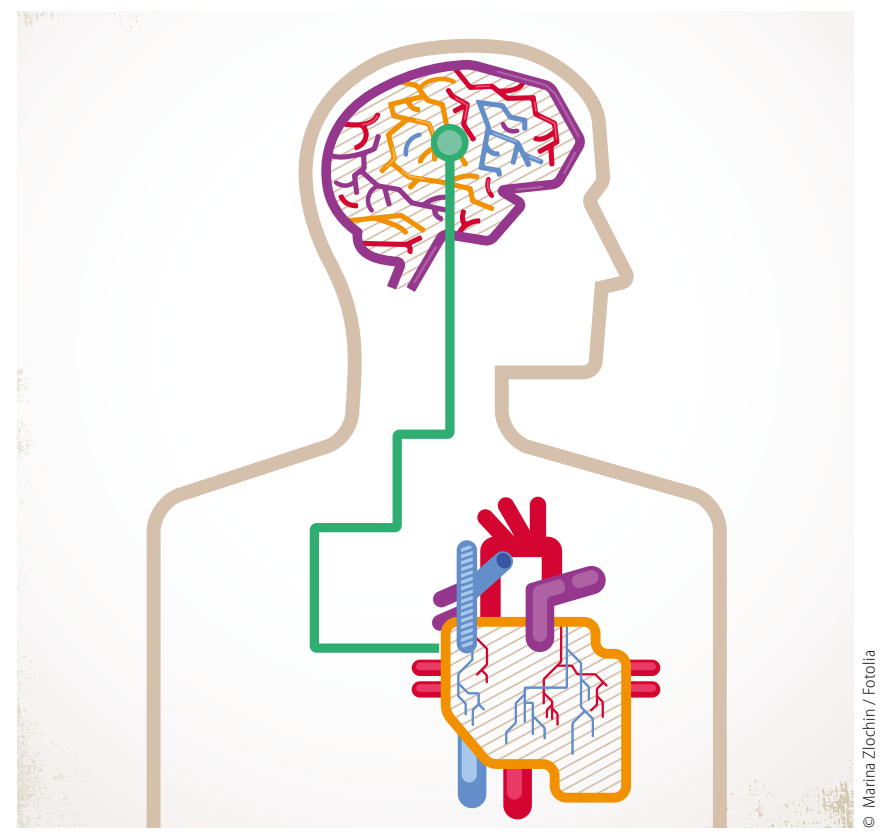

Bei KADN ist der autonome Draht vom Gehirn zum Herzen gestört.

potonien. Sie entstehen, wenn der Sympathikus auch betroffen ist und sind Zeichen einer fortgeschrittenen autonomen Neuropathie." Sie erläuterte, dass es zudem zu einer verminderten/fehlenden Wahrnehmung von Myokardischämien kommen kann.

Zur Basisdiagnostik bei diabetischer autonomer Neuropathie gehören die Messung der HFV bei Takt-Atmung (ExspirationInspiration-Quotient) und nach Lagewechsel (30:15-Quotient) sowie der Orthostasetest nach Schellong. Im Langzeit-EKG ist die relativ fixierte hohe Herzfrequenz laut Konz v.a. in den Nachtstunden ein Marker für die erhöhte kardiale Mortalität. „Die Verlängerung des QTc-Intervalls ist ein guter prognostischer Parameter der Mortalität bei Typ-1-Diabetes, bei Typ-2-Diabetes ist die fixierte Ruhefrequenz ein guter Marker Mortalität“, so Konz.

\section{Problem Hypoglykämie und KADN}

Über 50\% der Unterzucker bei Diabetes treten nachts auf, viele bleiben unbemerkt. Diabetiker im Alter über 60 Jahre haben eine erhöhte Hypoglykämiefrequenz, auch bei Nierenfschwäche steigt sie an. „Patienten mit KADN müssen Sie besonders vor Unterzucker schützen, denn sie haben auch schon in frühen Krankheitsstadien einen erhöhten Sympathikotonus“, so Konz. Dies gelte auch nachts, in der der Parasympathiko-Tonus physiologisch erhöht ist. Kämen dann noch Hypoglykämien hinzu, steige der Sympathikotonus noch weiter, was das Risiko für Herzrhythmusstörungen erhöhe.

Werner Zwick

Quelle: Symposium „Autonome Neuropathie bei Diabetes mellitus aus Sicht der Kardiologie“ am 19.2.16 in Unterschleißheim, Innere Medizin fachübergreifend - Diabetes grenzenlos", Veranstalter: Berufsverband Deutscher Internisten e.V. (BDI) und OmniaMed Deutschland GmbH. 